\title{
Evaluation of extrahepatic collateral arteries in hepatocellular carcinoma in three independent groups in a single center
}

\author{
YILIN ZHAO $^{1 *}$, ZHUTING FANG $^{2,3^{*}}$, JIANJUN LUO $^{3}$, QINGXIN LIU $^{3}$, \\ GANG XU $^{1}$, HENG PAN ${ }^{1}$, WEI WEI $^{1}$ and ZHIPING YAN ${ }^{3}$ \\ ${ }^{1}$ Department of Oncological and Vascular Interventional Radiology, Zhongshan Hospital, Xiamen University, Xiamen, \\ Fujian 361004; ${ }^{2}$ Department of Interventional Radiology, Provincial Hospital of Fujian, Fuzhou, Fujian 350014; \\ ${ }^{3}$ Department of Interventional Radiology, Zhongshan Hospital, Fudan University, Shanghai 200032, P.R. China
}

Received August 19, 2014; Accepted June 18, 2015

DOI: $10.3892 /$ etm.2015.2822

\begin{abstract}
To improve the efficacy of transcatheter arterial chemoembolization (TACE) for hepatocellular carcinoma (HCC), this study evaluated the prevalence and causes of extrahepatic arteries (EHAs) and identified feeding arteries in HCCs in three independent clinical groups in a single inverventional radiology center. Between November 2011 and September 2012, 942 cases of HCC were included in this retrospective study. The patients were treated in three independent groups of 285, 301 and 356 patients, respectively. Enhanced computed tomography, enhanced magnetic resonance imaging and digital subtraction angiography were reviewed retrospectively and correlations between the presence of tumor-feeding EHAs and tumor number, size and location in the liver, number of repeat TACE procedures and complications were assessed. There were 698 EHAs in the 942 cases of HCC, with 182, 233 and 283 EHAs in the three independent groups, respectively. Tumor size was associated with EHA formation; the percentages of patients with EHAs were 2.7 $\pm 3.0,5.5 \pm 0.5,43.2 \pm 4.0$, $61.8 \pm 5.2$ and $93.4 \pm 1.8 \%$ with tumor sizes of $2-3,3-5,5-7,7-9$ and $>9 \mathrm{~cm}$, respectively. There were $159 \pm 19$ EHAs in each group feeding tumors in peripheral locations in the liver, but only $48.7 \pm 6.8$ in the central zone. The most common EHA was the right inferior phrenic artery, with a mean of $101.0 \pm 14.1$ per group. The number of EHAs increased proportionally with the number of TACE sessions. The number of EHAs was positively associated with tumor size, peripheral location of the tumor and number of TACE sessions.
\end{abstract}

Correspondence to: Professor Zhiping Yan, Department of Interventional Radiology, Zhongshan Hospital, Fudan University, 180 Fenlin Road, Shanghai 200032, P.R. China

E-mail: yan.zhiping@zs-hospital.sh.cn

${ }^{*}$ Contributed equally

Key words: hepatocellular carcinoma, transcatheter arterial chemoembolization, extrahepatic arteries

\section{Introduction}

The normal liver obtains its blood supply from two sources, namely the portal vein, which provides $70 \%$ of the supply, and the hepatic artery, which provides $30 \%$ (1). However, primary liver cancer, also known as hepatoma or hepatocellular carcinoma (HCC), obtains blood exclusively from the hepatic artery (2,3). Since Yamada et al first reported transcatheter arterial embolization (TAE) treatment for HCC in 1983 (4), transcatheter arterial chemoembolization (TACE) has become widely used for the treatment of unresectable HCC, with the direct infusion of chemotherapeutic drugs to the tumor through the hepatic artery. The advantage of TACE is that greater concentrations of drug can be delivered directly to the tumor to reduce systemic toxicity and increase the efficacy of treatment $(5,6)$.

With the development of TACE, physicians have found that the main hepatic artery is not the only artery that supplies blood to hepatic tumors (7-9). Superselective TACE requires proper identification of tumor feeding arteries (10). Tumors are commonly supplied by extrahepatic collateral vessels, irrespective of the patency of the hepatic artery; indeed, most tumors fed from extrahepatic arteries (EHAs) are found in patients with a patent hepatic artery $(11,12)$. Investigators now consider that location of a tumor adjacent to the suspensory ligaments and bare area of the liver, direct invasion of or adhesion to adjacent organs, adhesions induced by previous abdominal surgery and recurrent tumor at the resection margin are the causes of EHA development (13-16).

In China, due to imbalances of economic development, many patients are diagnosed late with HCC and lose the opportunity for surgical treatment. TACE is an effective way to prolong the life of such patients. In practice, HCCs supplied by EHAs are often encountered even when the hepatic arteries are patent (17-19). Moreover, the development of EHAs interferes with effective treatment of the tumor with TACE. Although there are 401,000 patients in China diagnosed with liver cancer annually (20), few studies have focused on the formation of EHAs feeding hepatic tumors in Chinese patients.

The purpose of this study was to assess the occurrence of EHAs feeding HCCs, to determine the spectrum of extrahe- 
patic collateral vessels and the causes of EHA formation, and to study the imaging characteristics of EHAs on routine radiological examinations such as enhanced magnetic resonance imaging (MRI) and computed tomography (CT), to improve the efficacy and avoid complications in Chinese patients undergoing TACE of EHAs.

\section{Materials and methods}

Patients. This retrospective study was approved by the ethics committee of Zhongshan Hospital of Fudan University (Shanghai, China). Informed consent that the associated clinical information would possibly be used in future retrospective studies was obtained from all patients before TACE was performed. Each year, TACE is performed on $\sim 6,000$ patients from different regions of China in the Department of Interventional Radiology at Zhongshan Hospital of Fudan University. There are three completely independent clinical groups in this interventional radiological center, and each independent group treats $\sim 2,000$ TACE cases each year. Therefore, in this study the clinical data of the patients in these three independent clinical groups were compared to evaluate the extrahepatic collateral arteries in HCC and considered that the data may be representative of the occurrence of EHAs in Chinese patients with HCC.

A total of 942 patients were diagnosed with HCC by enhanced CT or MRI in the hospital from November 2011 to September 2012. The patients were treated in three independent groups of 285, 301 and 356 patients, respectively. These patients were underwent routine history taking, physical examination, blood tests, liver function and serum $\alpha$-fetoprotein (AFP) tests, ultrasonography, and abdominal enhanced CT or enhanced MRI. HCC was staged according to the Barcelona Clinic Liver Cancer (BCLC) criteria (21).

\section{Inclusion and exclusion criteria}

Inclusion criteria. The related clinical information of the patients treated with TACE was reviewed. Patients included in this retrospective study had unresectable BCLC stage B HCC, with $<50 \%$ tumor volume in the liver, Child stage A or B and a patent portal vein.

Exclusion criteria. Patients with BCLC stage B/C HCC with chronic renal failure, congestive heart failure, encephalopathy, previous upper gastrointestinal bleeding, severe coronary artery disease and portal vein occlusion were not included in this retrospective study.

Indications of EHAs in HCC. Enhanced CT or MRI was performed prior to TACE to confirm the location and size of tumors and the retention of iodized oil. Maximum tumor diameter was measured to indicate the tumor size. Results were evaluated by three radiologists. The levels of AFP, a tumor marker, were determined prior to TACE.

Certain findings suggested that it was necessary to search for EHAs by selective angiography. These were cases where: Tumors were in a peripheral or subcapsular location; there was exophytic tumor growth; a peripheral iodized-oil retention defect was exhibited within the tumor; a peripherally located portion of viable tumor was observed on a follow-up CT scan after TACE; hypertrophied arteries other than the hepatic artery were visible around the tumor on a CT scan; and a persistent elevation of the serum AFP level even after successful chemoembolization via the hepatic arteries.

Identification of arteries supplying tumors by initial TACE. Angiography or TACE was performed through the right femoral artery using a 4F or $5 \mathrm{~F}$ catheter and a 0.035 -inch J-shaped guide wire. Celiac axis arteriography was first undertaken to identify the location and number of tumors. Subsequently, selective cannulation was achieved by placing the catheter in the artery feeding the tumor.

In cases where celiac axis arteriography did not image the entire liver or there was a contrast staining defect in part of the liver, angiography of at least one of the superior mesenteric artery, inferior phrenic artery, omental branch, adrenal artery, intercostal artery, internal mammary artery, renal capsular artery, gastric artery or lumbar artery was conducted to determine the blood vessels supplying the tumor.

During TACE, in cases where the lipiodol emulsion did not cover the entire tumor or there was a defect in the tumor in addition to the deposition of lipiodol, other potential tumor supplying arteries were sought.

Chemoembolization. The emulsion comprised iodized oil (Lipiodol; Andre Guerbet, Aulnay-sous-Bois, France) and a chemotherapeutic drug, and was made by mixing, with stirring, 1-15 ml iodized oil and 10-30 mg doxorubicin hydrochloride or 50-150 mg oxaliplatin. The emulsion was then infused into the selected arteries. When the flow slowed or ceased and tiny portal venules were seen, TACE was stopped to avoid emulsion reflux and non-target embolization.

Follow-up for the presence of tumor-supplying EHAs. The patients underwent MRI or CT and measurement of AFP 4-6 weeks after TACE, to detect primary or recurrent tumors supplied by EHAs. In cases where the presence of a tumor other than the lipiodol-covered lesion or tumor recurrence was suspected, angiography was conducted to identify the EHAs supplying the tumor. Superselective cannulation using a microcatheter and microguide wire was performed to reach as far as possible into the target arteries and avoid non-target organ embolization. Chemoembolization of the EHAs in these patients was performed in the same manner as that through the hepatic artery. When sustained elevation of serum AFP was observed, even in the absence of tumor on MRI or CT, the presence of EHAs was suspected. In such cases, repeat TACE was performed to identify EHAs supplying a tumor.

Statistical analysis. All data are expressed as the mean \pm the standard error of the mean and $n$ represents the number of patients per clinical. Data processing and analysis were conducted using SPSS version 10.0 (SPSS Inc., Chicago, IL, USA). Statistical comparisons between the groups were performed using the rank sum test. Differences were considered significant at $\mathrm{P}<0.05$.

\section{Results}

Detection of EHAs. A total of 942 patients with HCC underwent TACE. In these patients, 698 EHAs were found in addition to 

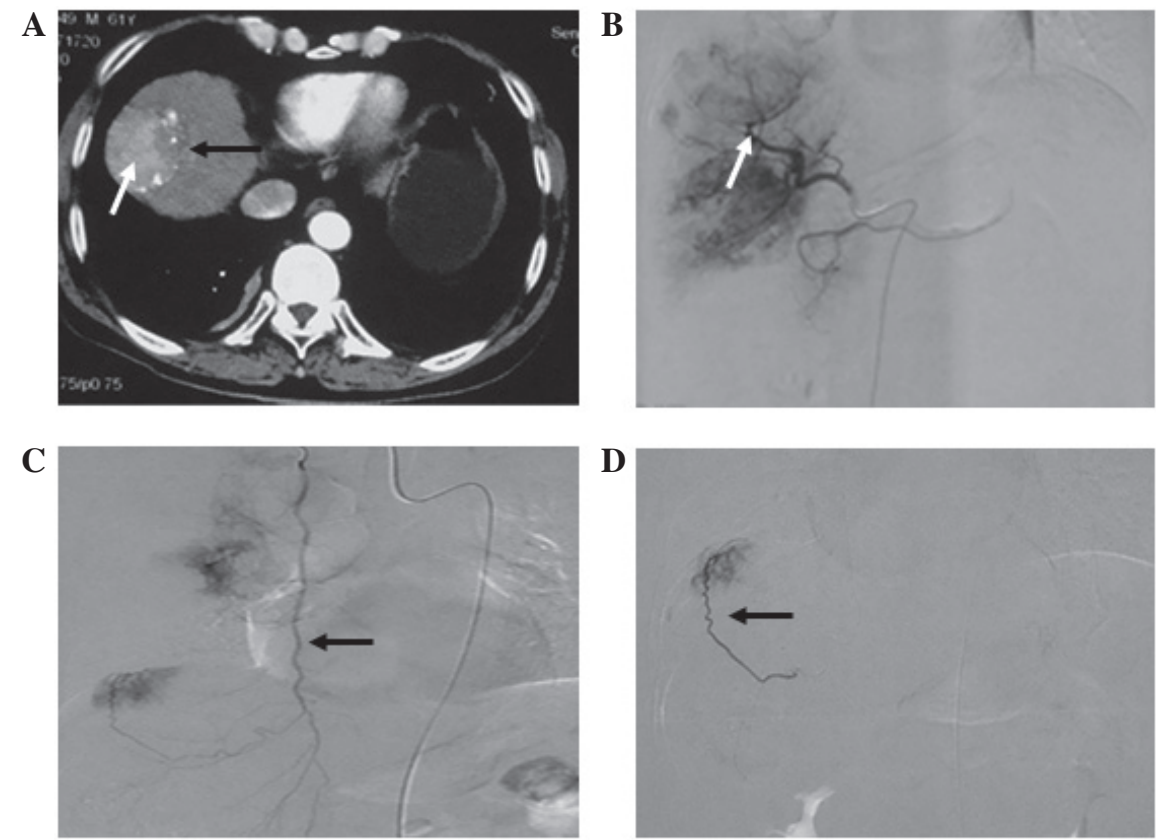

Figure 1. Hepatocellular carcinoma supplied by the right internal mammary artery. (A) Transverse computed tomography following transcatheter arterial chemoembolization shows an absence of iodized oil retention (black arrow) and iodized oil retention (white arrow). (B) Hepatic artery arteriogram (white arrow) shows an absence of staining in the upper part of the tumor. (C) Right internal mammary arteriogram (black arrow) shows tumor staining. (D) Right internal mammary arteriogram with a microcatheter inserted in the pericardiophrenic artery shows tumor staining (black arrow).

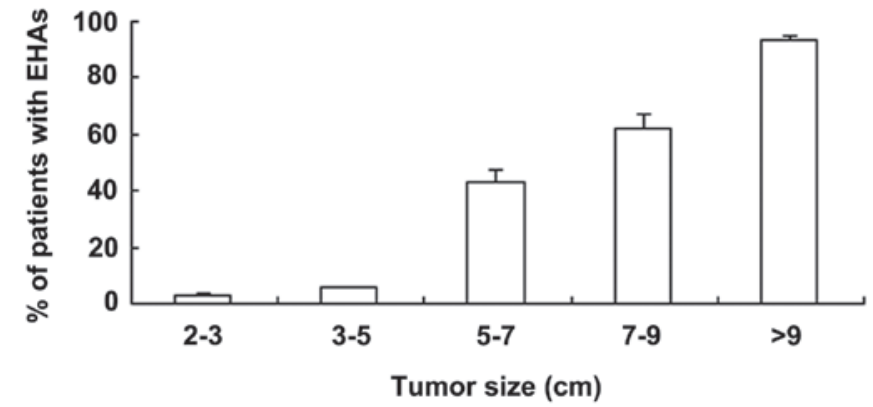

Figure 2. Prevalence of extrahepatic arteries (EHAs) supplying the hepatocellular carcinoma was closely associated with tumor size.

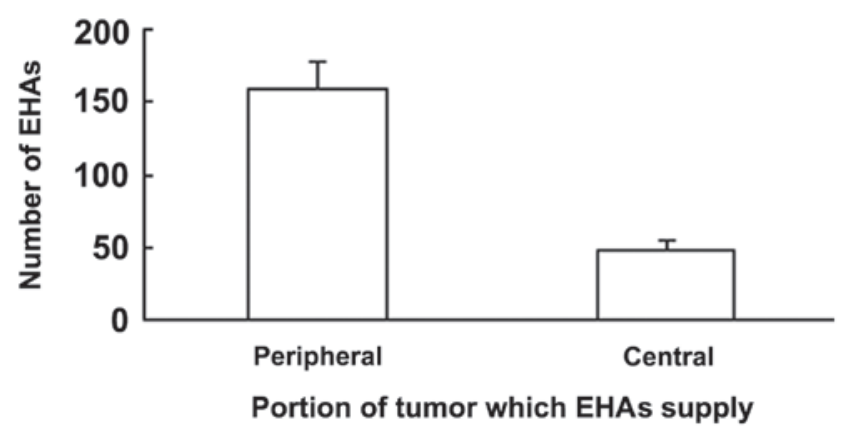

Figure 3. Most extrahepatic arteries (EHAs) supplied peripheral tumors.

the hepatic artery. The finding of elevated AFP following TACE usually implied that EHAs supplying the tumor had formed or existed. The presence of EHAs was often demonstrated by superselective angiography in addition to celiac artery angiography. In this study, $92.1 \%$ of EHAs were identified by arteriography and $7.9 \%$ were initially present in the $\mathrm{HCC}$ cases and were diagnosed by enhanced MRI or CT prior to angiography and chemoembolization (Fig. 1). In addition, $67.3 \%$ of patients, EHAs were indicated by persistantly increased levels of AFP. Hepatic artery occlusion is not a major cause of EHAs; in the present study, $84.5 \%$ of patients with EHAs had widely patent hepatic arteries.

Tumor size is associated with the formation of extrahepatic collateral arteries. The present study shows that the prevalence of an extrahepatic collateral supply to HCC was closely associated with tumor size. When the tumor was $<5 \mathrm{~cm}$ in diameter, the prevalence of EHAs at the initial TACE session was $<3.0 \pm 0.4 \%$; when the tumor was $>5 \mathrm{~cm}$ in diameter, the prevalence was $43.3 \pm 4.0 \%$. In patients with primary tumors $>5 \mathrm{~cm}$ in diameter, the majority of the EHAs fed the primary tumor.

The cumulative probability of EHAs in patients with a large tumor $(\geq 5 \mathrm{~cm})$ was significantly higher than that in those with a small tumor $(<5 \mathrm{~cm} ; \mathrm{P}<0.05)$. In patients who initially had a large primary tumor, EHAs supplying the primary tumor were usually present, whereas patients with a small tumor usually had EHAs supplying a recurrent tumor following several TACE sessions. The percentages of patients with EHAs were 2.7 \pm 3.0 , $5.5 \pm 0.5,43.2 \pm 4.0,61.8 \pm 5.2$ and $93.4 \pm 1.8 \%$ with tumors of $2-3$, 3-5, 5-7, 7-9 and $>9 \mathrm{~cm}$, respectively (Fig. 2). The difference between any two tumor size groups was significant $(\mathrm{P}<0.05)$.

Tumor location. There were $159 \pm 19$ EHAs feeding tumors in a peripheral location in the liver and $48.7 \pm 6.8$ feeding tumors in a central location. The number of the former was significantly higher than that of the latter $(\mathrm{P}<0.05 ;$ Fig. 3$)$. Tumors located in the bare area were found to be associated with a higher prevalence of EHAs than tumors not in the bare area. It was also 


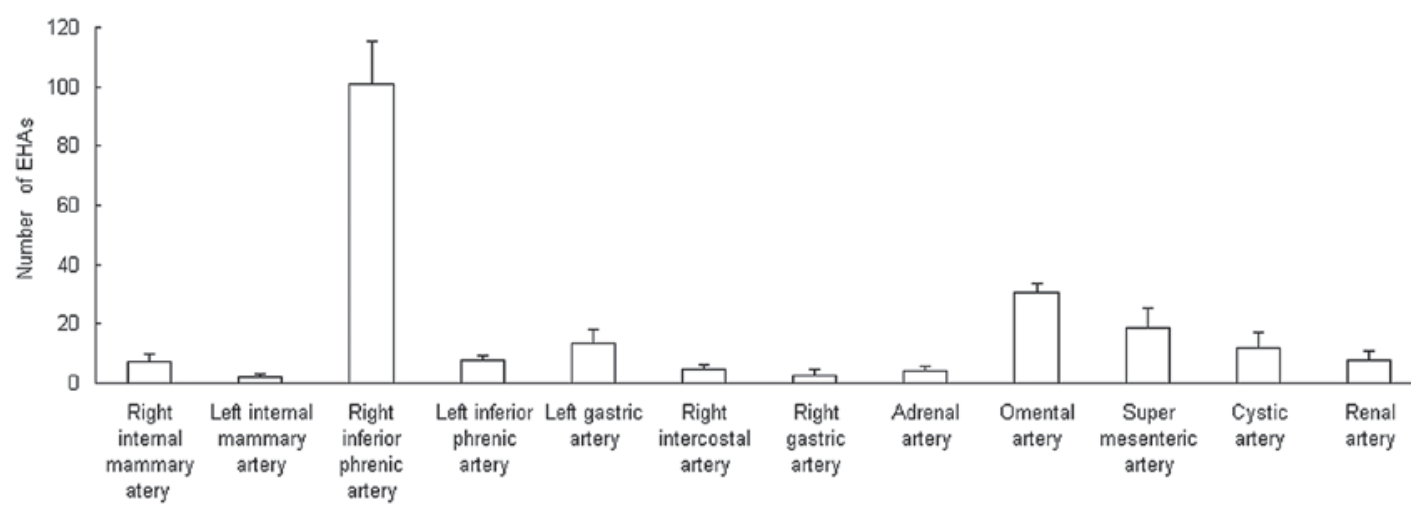

Figure 4. Extrahepatic arteries (EHAs) feeding tumors included the inferior phrenic artery, omental branch, adrenal artery, intercostal artery, internal mammary artery, renal capsular artery, gastric artery and lumbar artery.

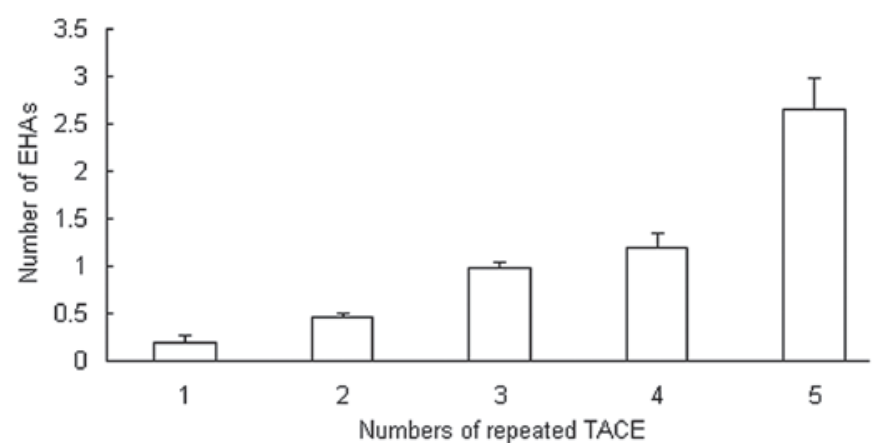

Figure 5. As the number of transcatheter arterial chemoembolization (TACE) sessions increased, the cumulative probability of extrahepatic arteries (EHAs) also increased.

observed that some tiny tumor foci located in the periphery of the liver were initially supplied by EHAs, and that EHAs supported local tumor progression as the primary tumor grew to reach a subcapsular location or exophytically invaded adjacent organs. The right inferior phrenic artery accounted for half of all of the observed EHAs and supplied the tumors in any liver location (Fig. 4).

Number of TACE sessions correlates with the cumulative probability of EHAs. It was observed that the majority of the EHAs supplied the recurrent small tumors $(<5 \mathrm{~cm})$ that appeared after repeated TACE. Peripheral hepatic artery attenuation or occlusion subsequent to multiple TACE sessions contributed to the development of EHAs. In the present study of 942 cases, EHAs were detected from the first to the fifth TACE session; $18.3 \pm 8.5 \%$ of the study subjects had EHAs at the initial session, $47.0 \pm 3.5 \%$ at the second session and $98.0 \pm 5.6 \%$ at the third session, and all had EHAs at the fourth and fifth sessions. In $46.1 \%$ of the 942 patients, EHAs supplied the primary tumors that were present at TACE. In the other patients, EHAs supplied recurrent tumors. The cumulative probability of the presence of EHAs is shown in Fig. 5. As the number of TACE sessions increased, the cumulative probability of EHAs also increased. From the initial TACE to the fifth TACE, the numbers of EHAs were $0.18 \pm 0.08,0.47 \pm 0.04,0.98 \pm 0.06,1.19 \pm 0.16$ and $2.66 \pm 0.31$, respectively (Fig. 5).
Adhesion and invasion. In $19.2 \%$ of the patients in this study, omental arteries were found to feed tumors that were adjacent to the stomach or colon and growing exophytically. The development of collateral vessels to a tumor could also be triggered by omental adhesion caused by exophytic growth, extracapsular HCC infiltration, abdominal postoperative omental or peritoneal adhesion, or recurrent tumor at the resection margin.

Types of EHA. The most common EHA was the inferior phrenic artery, with $101.0 \pm 14.1$ (Figs. 6-8), followed by the omental artery (30.3 \pm 3.1 ; Fig. 9) and adrenal artery (4.0 \pm 1.7 ; Figs. 7-8). Other EHAs included the right intercostal artery (4.7 \pm 1.5 ; Fig. 10), renal artery (7.7 \pm 3.1 ; Fig. 11), left inferior phrenic artery $(7.7 \pm 1.5)$, right internal mammary artery (7.3 \pm 2.5 ; Fig. 1), left gastric artery (13.3 \pm 4.6 ; Figs. 7 and 12), cystic artery (12.0 \pm 5.0 ; Fig. 13), super mesenteric artery (18.7 \pm 6.4 ; Fig. 14), right gastric artery $(2.3 \pm 2.3)$ and left internal mammary artery (2.0 \pm 1.0$)$, as shown in Fig. 4 .

Complications of EHA embolization. Complications were observed in 32 patients. Complications were usually associated with the location of the embolized lesions and included hiccup, acute gastroduodenal mucosal ischemic ulceration, cholecystitis, aggressive hepatic failure, hepatic artery spasm, hepatic artery constriction, hepatic artery occlusion, dissection, hepatic encephalopathy, left face-neck-shoulder pain, pleural effusion and lung infection.

\section{Discussion}

In this retrospective study, the radiological findings and imaging of HCCs supplied by EHAs were evaluated. The results demonstrated that the formation of EHAs in HCC is closely associated with the anatomical location of the tumor, tumor size, multiple TACE procedures, postsurgical adhesions and exophytic tumor growth. EHAs supplying HCCs require embolization; however, these arteries should be treated carefully and superselectively to avoid complications such as vascular dissection, spasm and non-target tissue or organ embolization.

There are various strategies to treat hepatic tumors by the transcatheter arterial approach (22-24). Familiarity with the blood supply that feeds hepatic tumors is essential not only to 

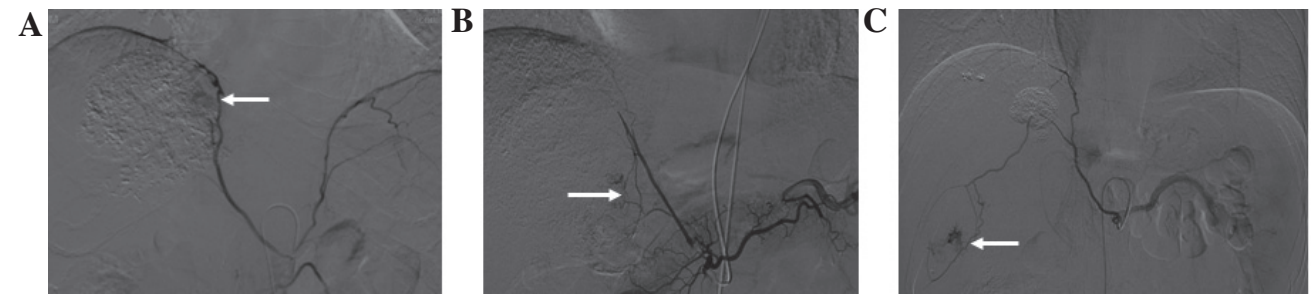

Figure 6. Hepatocellular carcinoma supplied by the right inferior phrenic artery. (A) Inferior phrenic arteriogram (arrow) indicates enhancement of tumor staining in S8 near the right diaphragm. (B) Inferior phrenic arteriogram indicates tumor staining in S5 (arrow). (C) Inferior phrenic arteriogram indicates tumor staining in S6 (arrow).
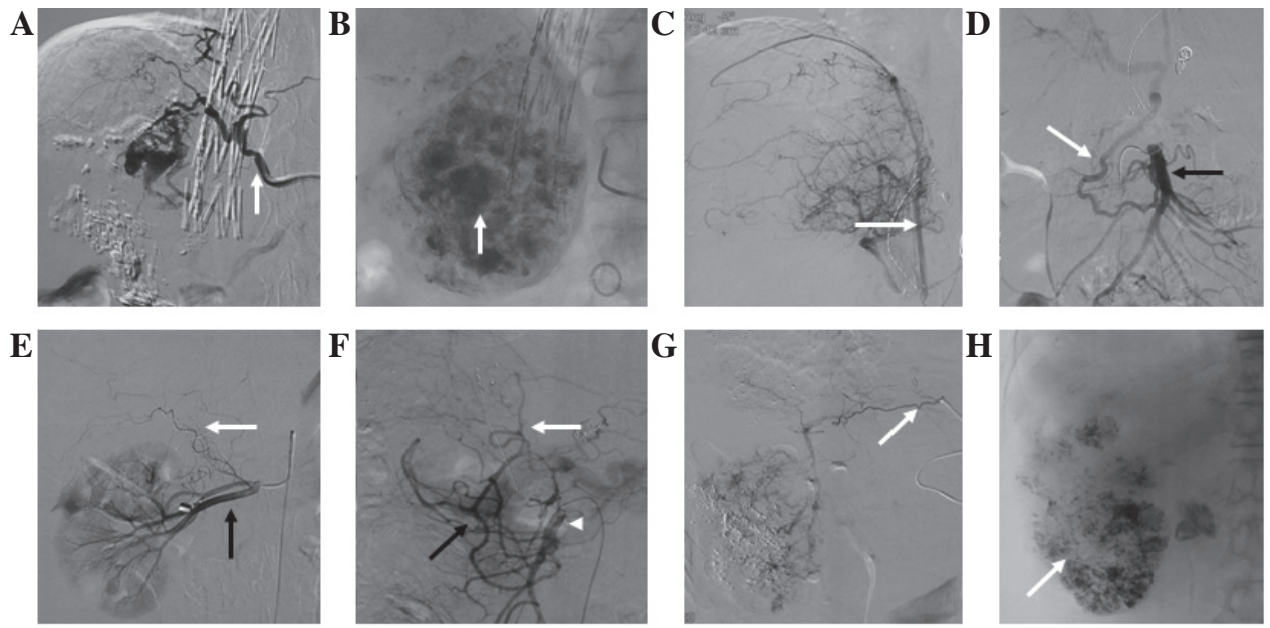

Figure 7. Development of extrahepatic arteries after several sessions of transcatheter arterial chemoembolization (TACE). (A) After the third session, the hepatic artery was occluded. The right inferior phrenic artery (arrow) supplied the tumor. (B) Embolization with iodized oil (arrow) through the right inferior phrenic artery. (C) The right inferior phrenic artery (arrow) supplied the tumor. (D) Hepatocellular carcinoma (HCC) supplied by a branch (white arrow) of the superior mesenteric artery (black arrow) after the fourth session of TACE. (E) The right adrenal artery (white arrow) supplied the partial tumor after the sixth session of TACE (black arrow indicates the renal artery). (F) HCC supplied by a branch (white arrow) from the pancreaticoduodenal arterial arch (black arrow; black arrow head indicates the superior mesenteric artery). (G) After the sixth session of TACE, the HCC was supplied by the left gastric artery (black arrow). (H) Embolization with iodized oil (white arrow) through the left gastric artery.
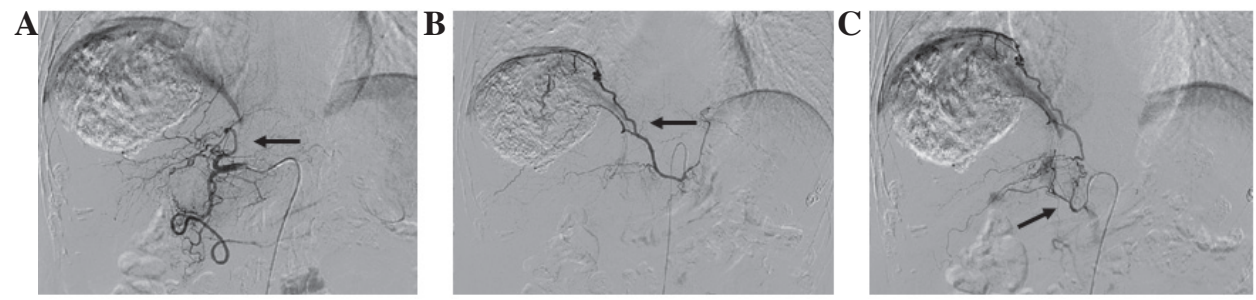

Figure 8. Hepatocellular carcinoma supplied by multiple arteries. (A) Celiac arteriogram shows that the hepatic artery (black arrow) supplied part of the tumor. (B) The inferior phrenic arteriogram (black arrow) supplied the upper part of the tumor. (C) Adrenal arteriogram (black arrow) shows that the adrenal artery also contributed to the tumor blood supply.

appreciate the limitations of these strategies but also to improve their therapeutic efficacy. The development of EHAs in HCC is a situation that limits the efficacy of TACE (25-27). In a study of TACE, Kim et al observed 2,104 extrahepatic collateral routes in 1,622 sessions in 860 patients and performed TACE via 1,556 extrahepatic collateral vessels in 732 patients (7).

Chemoembolization through EHAs can be attempted to improve the therapeutic efficacy of TACE. In this situation, selective catheterization should be achieved by manipulating the guide wire or catheter tip into the branches of the EHAs that are feeding the tumor, in order to prevent tumor progression or recurrence.
The main cause of EHAs was once considered to be hepatic artery occlusion by surgical ligation (which is no longer performed), mechanical injury or multiple TACE procedures. Certain studies have reported interruption or dissection of the hepatic artery by multiple TACE procedures to be the principal cause of the formation of extrahepatic collateral vessels $(28,29)$. A previous study showed that only $4 \%$ of patients with HCC suffered proximal hepatic artery occlusion and the majority of patients with a collateral supply had a patent hepatic artery (7).

The present study indicates a close association between the prevalence of an extrahepatic collateral supply to HCC and tumor size. It was found that the occurrence of an extrahepatic 

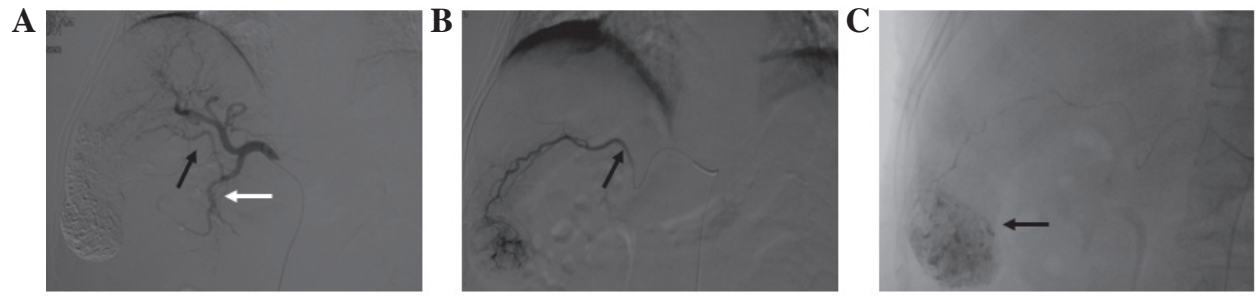

Figure 9. Hepatocellular carcinoma supplied by the omental branch of the gastroduodenal artery. (A) Celiac arteriogram shows hypervascular nodules in the liver supplied by a prominent omental branch (black arrow) of the gastroduodenal artery (white arrow). (B) Selective arteriogram shows staining of the tumor supplied by the omental branch (black arrow). (C) Complete iodized oil retention (black arrow) after transcatheter arterial chemoembolization through the omental branch of the gastroduodenal artery.
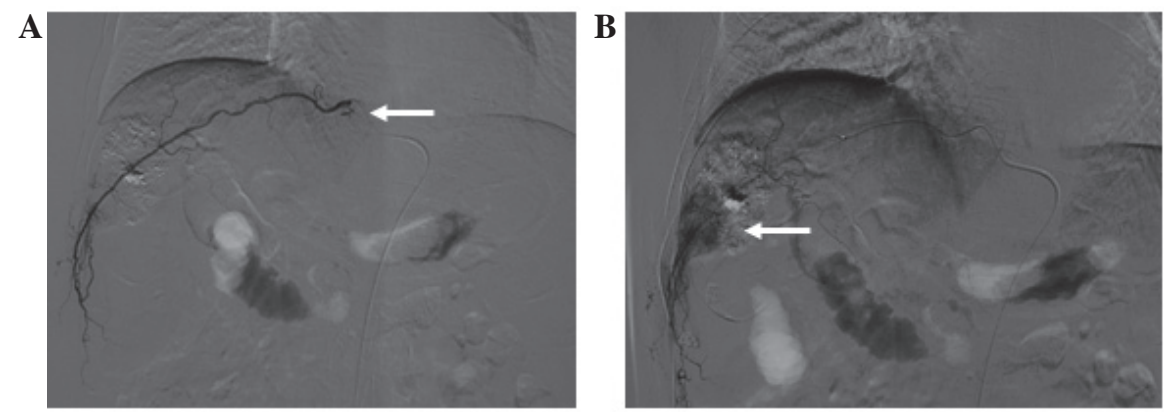

Figure 10. Hepatocellular carcinoma supplied by the intercostal artery. (A) Intercostal artery angiogram (white arrow) shows a hypervascular tumor of the liver. (B) Selective intercostal arteriogram with microcatheter inserted shows that the intercostal artery supplied the tumor (white arrow).
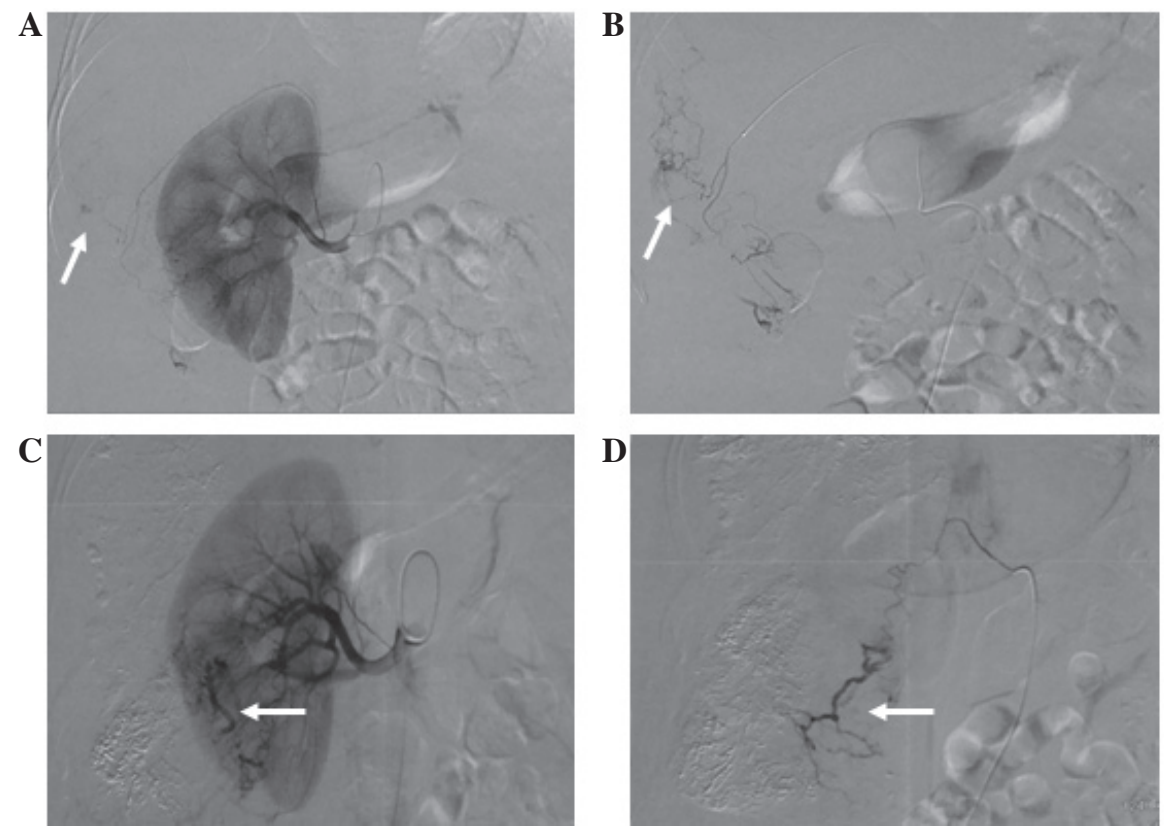

Figure 11. Hepatocellular carcinoma supplied by the renal artery. (A) Angiogram of a prominent superior renal capsular artery shows a hypervascular tumor (white arrow). (B) Renal arteriogram shows the hepatic tumor (white arrow) with a microcatheter inserted into the branch of the renal artery. (C) Right renal arteriogram shows staining of the tumor (white arrow) supplied by the renal artery. (D) A further selective angiogram shows tumor staining (white arrow).

collateral supply to HCC was closely associated with tumor size. These data are consistent with the literature (7-13).

The majority of the EHAs were found to supply the recurrent small $(<5 \mathrm{~cm})$ tumors that appeared after repeated TACE. Peripheral hepatic artery attenuation or occlusion following multiple TACE sessions contributed to the development of EHAs. In other studies, researchers have included only patients who underwent TACE $\leq 5$ times, due to the fact that patients treated with TACE $>5$ times had advanced disease and a poor prognosis $(7,13)$. As the number of TACE sessions increased, the cumulative probability of EHAs also increased, particularly for EHAs supplying recurrent tumors $(7,11)$.

It has been hypothesized that EHAs develop early at the bare area of the liver because the diaphragm and liver are 

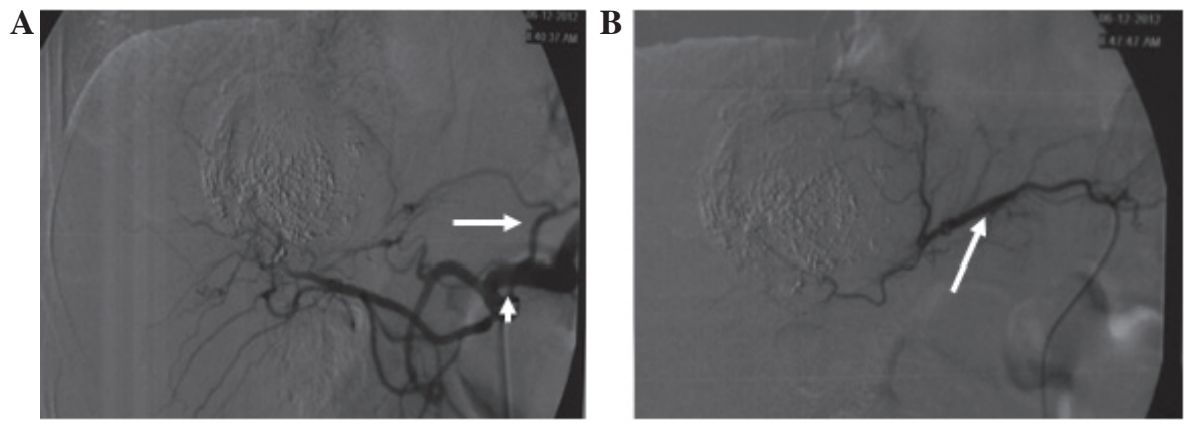

Figure 12. Hepatocellular carcinoma supplied by the left gastric artery. (A) Celiac arteriogram shows a viable tumor supplied by the left gastric artery (white arrow) with partial retention of iodized oil. (white arrow head indicates the hepatic artery). (B) Left gastric arteriogram (white arrow) shows tumor staining.

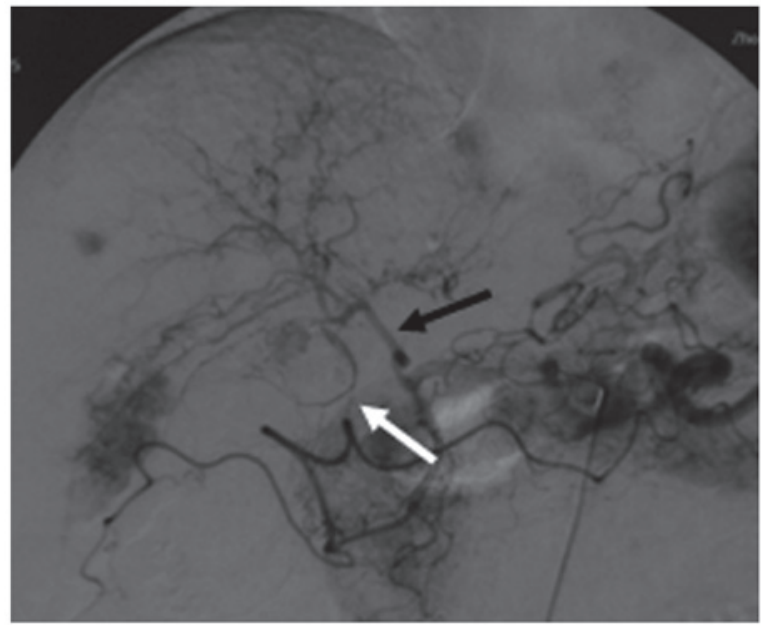

Figure 13. Hepatocellular carcinoma supplied by the cystic artery (white arrow) which is the first branch of the right hepatic artery (black arrow).

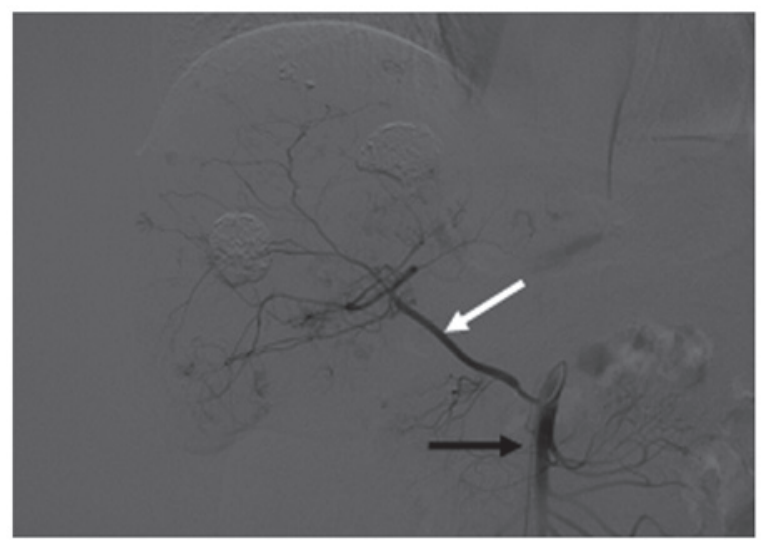

Figure 14. Hepatocellular carcinoma in the right hepatic lobe supplied by the right hepatic artery (white arrow) from the mesenteric superior artery (black arrow).

in direct contact without any capsular barrier, and the blood supply to the diaphragm can thus reach the liver by adherence (30). A surface tumor location is a prerequisite for and the most important factor associated with the formation of EHAs $(7,31)$. EHAs develop to supply the peripheral zone of the liver parenchyma, with the subsequent recurrence of tumor at remote sites in the peripheral zone supplied by the EHAs $(32,33)$. In the present study, it was found that tumors located in the bare area may be associated with a higher prevalence of EHAs than tumors not in the bare area.

The development of collateral vessels to a tumor could be triggered by omental adhesion caused by exophytic growth, extracapsular HCC infiltration, abdominal postoperative omental or peritoneal adhesion, or recurrent tumor at the resection margin. In cases of multiple TACE, hepatic infarction in peripheral zones could trigger omental or peritoneal adhesion leading to the development of extrahepatic collateral arteries (34). Direct contact with or invasion into other organs, including the stomach (35), colon (36), adrenal gland (37) and kidney (38), may create extrahepatic collateral arteries to the tumor from these organs.

The inferior phrenic artery (29), omental branch (38), adrenal artery, intercostal artery, internal mammary artery, renal capsular artery (39), left gastric artery (40), gastroduodenal artery, cystic artery and mesenteric superior artery are among the EHAs known to feed tumors (41). Considering the broad contact between the liver and the diaphragm, it may be expected that diaphragmatic blood supplies, including the inferior phrenic, internal mammary and intercostal arteries, are major sources of collateral circulation.

When collateral vessels are chemoembolized, there is a risk of embolizing non-target tissues or organs. Hiccups were usually the result of embolization through right inferior phrenic arteries (42). Acute gastroduodenal mucosal ischemic ulceration or necrosis typically occurs following embolization through the branches of the gastroduodenal artery (43). Cholecystitis is frequently caused by the embolization of the cystic artery (44). Aggressive hepatic failure may occur as a result of multiple embolization through the hepatic artery and EHA branches (45). Hepatic artery spasm, hepatic artery constriction and hepatic artery occlusion are typically caused by the repeated stimulation of the hepatic artery with a $5 \mathrm{~F}$ catheter or by careless actions (46). Pleural effusion is common when tumors adjacent to the diaphragm are treated (47). Lung infection usually results from an overdose of the iodized oil infusion (48).

Strategies to avoid these complications include superselective catheterization of the specific branch supplying the tumor, avoiding reflux of embolization material into non-target vessels, and use of coils and microparticles to occlude normal vessels before chemoembolization is performed (49). However, the risk in patients with advanced HCC is worthwhile if the EHAs of tumors are carefully occluded with a microcatheter. 
Selective angiography of EHAs was performed in patients in whom it was suspected that there was a blood supply to a tumor. However, unsuspected EHAs may have been missed in some patients, because some tumor-feeding arteries are difficult to identify as a result of insufficient tumor vascularity or overlapping vessels. The development of alternative methods for identifying EHAs, in addition to routine examinations such as MRI, enhanced CT and digital subtraction angiography, is required. In this study, it was not possible to chemoembolize every detected extrahepatic collateral vessel because of potential complications or difficulties in superselective catheterization. In cases where the EHA supply could not be accessed, the tumor lesion was treated with local ethanol injection or radiofrequency ablation guided with sonography or CT.

In conclusion, the number of extrahepatic collateral arteries feeding tumors was positively associated with tumor size, peripheral location of the tumor in the liver, and the number of TACE sessions. In addition to the main hepatic artery, the most common EHAs were the right inferior phrenic artery and the omental artery. Knowledge of changes in the hepatic artery can help when performing repeated TACE and reduce the time required for the procedure, and may help to improve the outcome of patients with HCC.

\section{References}

1. Sergio A, Cristofori C, Cardin R, et al: Transcatheter arterial chemoembolization (TACE) in hepatocellular carcinoma (HCC): The role of angiogenesis and invasiveness. Am J Gastroenterol 103: 914-921, 2008.

2. Lee HS, Kim JS, Choi IJ, et al: The safety and efficacy of transcatheter arterial chemoembolization in the treatment of patients with hepatocellular carcinoma and main portal vein obstruction. A prospective controlled study. Cancer 79: 2087-2094, 1997.

3. Takayasu K, Arii S, Ikai I, et al: Prospective cohort study of transarterial chemoembolization for unresectable hepatocellular carcinoma in 8510 patients. Gastroenterology 131: 461-469, 2006

4. Yamada R, Sato M, Kawabata M, et al: Hepatic artery embolization in 120 patients with unresectable hepatoma. Radiology 148: 397-401, 1983

5. Yoon CJ, Chung JW, Park JH, et al: Transcatheter arterial chemoembolization with paclitaxel-lipiodol solution in rabbit VX2 liver tumor. Radiology 229: 126-131, 2003.

6. Tsimberidou AM, Letourneau K, Fu S, et al: Phase I clinical trial of hepatic arterial infusion of paclitaxel in patients with advanced cancer and dominant liver involvement. Cancer Chemother Pharmacol 68: 247-253, 2011

7. Kim HC, Chung JW, Lee W, Jae HJ and Park JH: Recognizing extrahepatic collateral vessels that supply hepatocellular carcinoma to avoid complications of transcatheter arterial chemoembolization. Radiographics 25 (Suppl 1): S25-S39, 2005.

8. Miyayama S, Matsui O, Taki K, et al: Extrahepatic blood supply to hepatocellular carcinoma: Angiographic demonstration and transcatheter arterial chemoembolization. Cardiovasc Intervent Radiol 29: 39-48, 2006.

9. Park SI, Lee DY, Won JY and Lee JT: Extrahepatic collateral supply of hepatocellular carcinoma by the intercostal arteries. J Vasc Interv Radiol 14: 461-468, 2003.

10. Shibata T, Kojima N, Tabuchi T, Itoh $\mathrm{K}$ and Konishi J: Transcatheter arterial chemoembolization through collateral arteries for hepatocellular carcinoma after arterial occlusion. Radiat Med 16: 251-256, 1998.

11. Chung JW, Kim HC, Yoon JH, et al: Transcatheter arterial chemoembolization of hepatocellular carcinoma: Prevalence and causative factors of extrahepatic collateral arteries in 479 patients. Korean J Radiol 7: 257-266, 2006.

12. Wang YL, Li MH, Cheng YS, Shi HB and Fan HL: Influential factors and formation of extrahepatic collateral artery in unresectable hepatocellular carcinoma. World J Gastroenterol 11: 2637-2642, 2005.
13. Kim HC, Chung JW, Park JH, et al: Transcatheter arterial chemoembolization for hepatocellular carcinoma: Prospective assessment of the right inferior phrenic artery with C-arm CT. J Vasc Interv Radiol 20: 888-895, 2009.

14. Kim HC, Chung JW, Jae HJ, et al: Hepatocellular carcinoma: Prediction of blood supply from an internal mammary artery with multi-detector row CT. J Vasc Interv Radiol 19: 1419-1426, 2008.

15. Kim HC, Chung JW, Choi SH, et al: Internal mammary arteries supplying hepatocellular carcinoma: Vascular anatomy at digital subtraction angiography in 97 patients. Radiology 242: 925-932, 2007.

16. Kim HC, Chung JW, Jae HJ, et al: Hepatocellular carcinoma: Transcatheter arterial chemoembolization of the gonadal artery. J Vasc Interv Radiol 17: 703-709, 2006.

17. Peng ZW, Guo RP, Zhang YJ, et al: Hepatic resection versus transcatheter arterial chemoembolization for the treatment of hepatocellular carcinoma with portal vein tumor thrombus. Cancer 118: 4725-4736, 2012.

18. Xi T, Lai EC, Min AR, et al: Adjuvant transarterial chemoembolization after curative resection of hepatocellular carcinoma: A non-randomized comparative study. Hepatogastroenterology 59: 1198-1203, 2012.

19. Luo XJ, Tan WF, Yi B, et al: Surgery of hepatocellular carcinoma complicated with cancer thrombi in bile duct: Efficacy for criteria for different therapy modalities. Langenbecks Arch Surg 394: 1033-1039, 2009.

20. Wu Q and Qin SK: Features and treatment options of Chinese hepatocellular carcinoma. Chin Clin Oncol 2: 38, 2013.

21. Llovet JM, Fuster J and Bruix J; Barcelona-Clínic Liver Cancer Group: The Barcelona approach: Diagnosis, staging, and treatment of hepatocellular carcinoma. Liver Transpl 10 (2 Suppl 1): S115-S120, 2004.

22. Lencioni R, Chen XP, Dagher L and Venook AP: Treatment of intermediate/advanced hepatocellular carcinoma in the clinic: How can outcomes be improved? Oncologist 15 (Suppl 4): 42-52, 2010.

23. Forner A, Reig ME, de Lope CR and Bruix J: Current strategy for staging and treatment: The BCLC update and future prospects. Semin Liver Dis 30: 61-74, 2010.

24. Welker MW, Zangos S, Kriener S, et al: Sequential therapy of transarterial chemoembolisation and sorafenib in intermediate stage hepatocellular carcinoma. J Gastrointest Cancer 41: 149-152, 2010.

25. Miyayama S, Yamashiro M, Okuda M, et al: The march of extrahepatic collaterals: Analysis of blood supply to hepatocellular carcinoma located in the bare area of the liver after chemoembolization. Cardiovasc Intervent Radiol 33: 513-522, 2010.

26. Won JY, Lee DY, Lee JT, et al: Supplemental transcatheter arterial chemoembolization through a collateral omental artery: Treatment for hepatocellular carcinoma. Cardiovasc Intervent Radiol 26: 136-140, 2003.

27. Kimura S, Okazaki M, Higashihara $\mathrm{H}$, et al: Clinico-roentogenologic findings in hepatocellular carcinoma fed by the right inferior phrenic artery at the initial chemoembolization. Hepatogastroenterology 56: 191-198, 2009.

28. Vaidya S, Dighe M, Bhargava P and Dick AA: Chronic hepatic artery occlusion with collateral formation: Imaging findings and outcomes. Transplant Proc 43: 1770-1776, 2011.

29. Yang L, Zhang XM, Ren YJ, Miao ND, Huang XH and Dong GL: The features of extrahepatic collateral arteries related to hepatic artery occlusion and benefits in the transarterial management of liver tumors. Radiol Res Prac 2013: 535272, 2013.

30. Miyayama S, Yamashiro M, Yoshie Y, et al: Inferior phrenic arteries: Angiographic anatomy, variations and catheterization techniques for transcatheter arterial chemoembolization. Jpn J Radiol 28: 502-511, 2010.

31. Guan YS, Zheng XH, Zhou XP, et al: Multidetector CT in evaluating blood supply of hepatocellular carcinoma after transcatheter arterial chemoembolization. World J Gastroenterol 10: 2127-2129, 2004.

32. Kodama Y, Shimizu T, Endo H, et al: Spontaneous rupture of hepatocellular carcinoma supplied by the right renal capsular artery treated by transcatheter arterial embolization. Cardiovasc Intervent Radiol 25: 137-140, 2002.

33. Eurvilaichit C: Outcome of transcatheter oily chemoembolization in patients with hepatocellular carcinoma. Hepatogastroenterology 51: 20-24, 2004. 
34. Paul SB, Gamanagatti SR, Mukund A, Abbas SZ and Acharya SK: Transarterial chemoembolization for hepatocellular carcinoma: significance of extrahepatic collateral supply. Indian J Cancer 48: 339-344, 2011.

35. Hu ML, Tai WC, Chuah SK, et al: Gastric metastasis of hepatocellular carcinoma via a possible existing retrograde hematogenous pathway. J Gastroenterol Hepatol 25: 408-412, 2010.

36. Zech CJ, Bilzer M, Mueller-Lisse UG, et al: Perforation of the colon: A rare complication of hepatocellular carcinoma. Acta Radiol 47: 538-542, 2006.

37. Uchino K, Tateishi R, Shiina S, et al: Hepatocellular carcinoma with extrahepatic metastasis: Clinical features and prognostic factors. Cancer 117: 4475-4483, 2011.

38. Choi JW, Kim HC, Chung JW, et al: Chemoembolization via branches from the splenic artery in patients with hepatocellular carcinoma. Cardiovasc Intervent Radiol 35: 90-96, 2012.

39. Baba Y, Miyazono N, Inoue H, et al: Small hepatocellular carcinoma supplied by the right renal capsular artery. A case report. Acta Radiol 40: 449-450, 1999.

40. Jeon UB, Lee JW, Baik SK, et al: Hepatocellular carcinoma supplied from the short gastric artery: Treatment with chemoembolization. Cardiovasc Intervent Radiol 35: 1512-1514, 2012.

41. Miyayama S, Yamashiro M, Okuda M, et al: Hepatocellular carcinoma supplied by the right lumbar artery. Cardiovasc Intervent Radiol 33: 53-60, 2010.

42. Shin SW, Do YS, Choo SW, et al: Diaphragmatic weakness after transcatheter arterial chemoembolization of inferior phrenic artery for treatment of hepatocellular carcinoma. Radiology 241: 581-588, 2006.
43. Nakamura H, Hashimoto T, Oi H, Sawada S and Furui S: Prevention of gastric complications in hepatic arterial chemoembolization. Balloon catheter occlusion technique. Acta Radiol 32: 81-82, 1991.

44. Shah RP and Brown KT: Hepatic arterial embolization complicated by acute cholecystitis. Semin Intervent Radiol 28: 252-257, 2011.

45. Jeon SH, Park KS, Kim YH, et al: Incidence and risk factors of acute hepatic failure after transcatheter arterial chemoembolization for hepatocellular carcinoma. Korean J Gastroenterol 50: 176-182, 2007 (In Korean).

46. Sueyoshi E, Hayashida T, Sakamoto I and Uetani M: Vascular complications of hepatic artery after transcatheter arterial chemoembolization in patients with hepatocellular carcinoma. AJR Am J Roentgenol 195: 245-251, 2010.

47. Negrini S, Zoppoli G, Andorno E, Picciotto A and Indiveri F: Iodized oil pleural effusion in a patient previously treated with transarterial chemoembolization for hepatocellular carcinoma. Chest 138: 193-195, 2010.

48. Naorungroj T, Naksanguan T and Chinthammitr Y: Pulmonary lipiodol embolism after transcatheter arterial chemoembolization for hepatocellular carcinoma: A case report and literature review. J Med Assoc Thai 96 (Suppl 2): 270-275, 2013.

49. Poggi G, Pozzi E, Riccardi A, et al: Complications of image-guided transcatheter hepatic chemoembolization of primary and secondary tumours of the liver. Anticancer Res 30: 5159-5164, 2010 . 